Article

\title{
Overexpression of the Panax ginseng CYP703 Alters Cutin Composition of Reproductive Tissues in Arabidopsis
}

\author{
Jihyun Kim ${ }^{1,+} \oplus$, Jeniffer Silva ${ }^{2,+}$, Chanwoo Park ${ }^{1}$, Younghun Kim ${ }^{1}$, Nayeon Park ${ }^{1}$, Johan Sukweenadhi ${ }^{3}$, \\ Junping Yu ${ }^{4}$, Jianxin Shi ${ }^{5}{ }^{\circledR}$, Dabing Zhang ${ }^{5}$, Keun Ki Kim ${ }^{1}$, Hong-Joo Son ${ }^{1}{ }^{1}$, Hyeon Cheal Park ${ }^{1}$, \\ Chang-Oh Hong ${ }^{1}$, Kwang Min Lee ${ }^{1}$ (D) and Yu-Jin Kim ${ }^{1, * \mathbb{D}}$
}

check for

updates

Citation: Kim, J.; Silva, J.; Park, C.; Kim, Y.; Park, N.; Sukweenadhi, J.; Yu, J.; Shi, J.; Zhang, D.; Kim, K.K.; et al. Overexpression of the Panax ginseng CYP703 Alters Cutin Composition of Reproductive Tissues in Arabidopsis. Plants 2022, 11, 383. https://doi.org/10.3390/ plants11030383

Academic Editor: Baohong Zhang

Received: 24 December 2021

Accepted: 28 January 2022

Published: 30 January 2022

Publisher's Note: MDPI stays neutral with regard to jurisdictional claims in published maps and institutional affiliations.

Copyright: (c) 2022 by the authors Licensee MDPI, Basel, Switzerland. This article is an open access article distributed under the terms and conditions of the Creative Commons Attribution (CC BY) license (https:// creativecommons.org/licenses/by/ $4.0 /)$.
1 Department of Life Science and Environmental Biochemistry, Life and Industry Convergence Research Institute, Pusan National University, Miryang 50463, Korea; jihyun0_0@pusan.ac.kr (J.K.); musgdmu8869@naver.com (C.P.); edward69543@naver.com (Y.K.); xosk126@naver.com (N.P.); kkkim@pusan.ac.kr (K.K.K.); shjoo@pusan.ac.kr (H.-J.S.); hcpark@pusan.ac.kr (H.C.P.); soilchem@pusan.ac.kr (C.-O.H.); leekm@pusan.ac.kr (K.M.L.)

2 Department of Research and Development, The Bridge Biofoundry, Ciudad de Saber, Clayton 0843-03081, Panama; jeniffersilva.yat@gmail.com

3 Department of Plant Biotechnology, Faculty of Biotechnology, Universitas Surabaya, Raya Kalirungkut, Kalirungkut, Surabaya 60294, East Java, Indonesia; sukweenadhi@gmail.com

4 Key Laboratory of Biotechnology Shaanxi Province, College of Life Sciences, Chinese Education Ministry's Key Laboratory of Western Resources and Modern Biotechnology, Northwest University, Xi'an 710069, China; yujunping521@sina.com

5 Joint International Research Laboratory of Metabolic and Developmental Sciences, School of Life Sciences and Biotechnology, Shanghai Jiao Tong University, Shanghai 200240, China; jianxin.shi@sjtu.edu.cn (J.S.); zhangdb@sjtu.edu.cn (D.Z.)

* Correspondence: yjkim2020@pusan.ac.kr; Tel.: +82-055-350-5546

$\dagger$ These authors contributed equally to this work.

\begin{abstract}
Cytochrome P450 (CYP) catalyzes a wide variety of monooxygenation reactions in plant primary and secondary metabolisms. Land plants contain CYP703, belonging to the CYP71 clan, which catalyzes the biochemical pathway of fatty acid hydroxylation, especially in male reproductive tissues. Korean/Asian ginseng (Panax ginseng Meyer) has been regarded as one of important medicinal plant for a long time, however the molecular mechanism is less known on its development. In this study, we identified and characterized a CYP703A gene in P. ginseng (PgCYP703A4), regarding reproductive development. $\mathrm{PgCYP703A4}$ shared a high-sequence identity (81-83\%) with predicted amino acid as CYP703 in Dancus carota, Pistacia vera, and Camellia sinensis as well as $76 \%$ of amino acid sequence identity with reported CYP703 in Arabidopsis thaliana and 75\% with Oryza sativa. Amino acid alignment and phylogenetic comparison of $P$. ginseng with higher plants and known $A$. thaliana members clearly distinguish the CYP703 members, each containing the AATDTS oxygen binding motif and PERH as a clade signature. The expression of PgCYP704B1 was only detected in P. ginseng flower buds, particularly in meiotic cells and the tapetum layer of developing anther, indicating the conserved role on male reproduction with At- and Os- CYP703. To acquire the clue of function, we transformed the PgCYP703A4 in A. thaliana. Independent overexpressing lines (PgCYP703A4ox) increased silique size and seed number, and altered the contents of fatty acids composition of cutin monomer in the siliques. Our results indicate that $\mathrm{PgCYP703A4}$ is involved in fatty acid hydroxylation which affects cutin production and fruit size.
\end{abstract}

Keywords: cytochrome P450; reproductive tissues; PgCYP703A4; fatty acid; reproduction; Panax ginseng

\section{Introduction}

The cytochrome P450 (CYP) superfamily of enzymes, which catalyze diverse substrates through oxygenation and hydroxylation reactions, are found in all organisms [1]. Plant CYPs are involved in a variety of biochemical pathways that produce primary and 
secondary metabolites, which constitute one of the largest families of enzymes in higher plants [2]. The diversification of land plant CYP families emerges during flowering plant evolution and specializes plant species with unique reactions [3]. The identification of CYP genes aids in understanding the evolution of various groups of enzymes and their conserved and diversified functions.

The CYP703 gene family was found across the land plant taxa, suggesting that it encodes an essential function [4]. Land plants developed specialized cell layer to adapt to the environment, including cutin synthesis. As cutin cover in most plant organ of land plant, male reproductive organ of flowering plants is covered with a hydrophobic polymer barrier derived from fatty acids. The production of functional gametophytes leads to a successful proliferation in flowering plants [5]. Male reproduction is a complex and highly coordinated biological process that includes the development of the male reproductive organ: the stamen that contains the microspores and pollen. The study of male reproduction is important not only for increasing crop yield but for producing improved crops, such as superhybrid plants, through the use of male sterile lines [6].

The CYP703 family is involved in the in-chain hydroxylation of mid-chain fatty acids, which is essential for the biopolymers of pollen exine, the outer wall of a pollen grain [7,8], and cutin, one of the most abundant lipid polymers and an important adaptive trait of plants to their terrestrial environment [9]. A. thaliana CYP703A2 (AtCYP703A2) catalyzes the conversion of medium-chain saturated fatty acids to the corresponding monohydroxylated fatty acids, with a preferential hydroxylation of lauric acid at the C-7 position [8]. In comparison, Oryza sativa CYP703A3 (OsCYP703A3) can only catalyze in-chain hydroxylation of lauric acid (C12), preferentially at position 7 [7]. The A. thaliana mutant, cyp703a2, exhibits impaired pollen development and a partial male sterile phenotype due to the lack of an exine [8]. O. sativa CYP703A3 resulted in a complete male sterility with an abnormal anther epidermis, as well as defective pollen exine, indicating that the diversified function of CYP703A during evolution [7]. Both participated in a conserved pathway of in-chain hydroxylation of lauric acid that is required for male reproductive development. Some male-specific gene sequences that determine sex, where CYP703 was found, have been identified in the Phoenix tree which belongs to dioecious species. [10]. Further studies in other plants will help elucidate the diversified CYP703 function of fatty acid hydroxylation in plant reproductive development.

Panax ginseng is a perennial herb that has been cultivated for its highly valued root for medicinal purposes [11]. P. ginseng typically starts its reproduction at the third year of growth $[12,13]$. Attempts to increase the yield of $P$. ginseng and ginsenosides have been conducted by developing P. ginseng hybrids, and although they display heterosis, F1 hybrid plants exhibited male sterility that derived from pollen defects at the young microspore stage [13]. We previously studied and described the morphogenesis of the anther and carpel at a cytological level to understand and specify the reproductive developmental phases of $P$. ginseng $[12,14]$ and identified the gene expression of $P g C Y P 703$ in the anther tapetum layer [12]. Despite the importance of $P$. ginseng reproductive development, studies on functional gene analysis and molecular regulation remain scarce. In the studies presented here, we isolated and cloned the CYP703A gene from P. ginseng, PgCYP703A, which is highly expressed in flower buds during anther development. Surprisingly, in $A$. thaliana, overexpression of $\mathrm{PgCY}$ P704B1 resulted in the enhanced in silique length, in terms of fruit size, which is potentially caused by the alteration of saturated fatty acids and hydroxy fatty acids in siliques.

\section{Material and Methods}

\subsection{Plant Materials and Growth Conditions}

The ginseng ( $P$. ginseng Mayer) plant organs (root body, stem, leaf, flower bud, and fruit) were obtained from hydroponically cultured ginseng. Columbia ecotype (CS60000) of A. thaliana was used for gene overexpression. Sterilized seeds were sown on half-strength Murashige and Skoog medium (Duchefa Biochemie) containing 1\% sucrose, $0.8 \%(w / v)$ 
agar, and $\mathrm{pH}$ 5.7. Three-day-old cold-treated seeds were germinated under long-day photoperiods of 16 -h light $/ 8$-h dark at $23^{\circ} \mathrm{C}$. The transformants were screened on hygromycin $(50-\mu \mathrm{g} / \mathrm{mL})$-selective medium plates. Ten-day-old seedlings were then transplanted to soil and cultivated for five weeks under the same light/dark conditions [15].

\subsection{Identification of PgCYP703A4 Gene and Sequence Analysis}

To obtain a coding sequence (CDS) of the PgCYP703A4 gene, homologous sequences of CYP703 was obtained based on A. thaliana sequence by homology-based PCR from P. ginseng flower cDNA. A complete genomic DNA sequence was obtained and analyzed from the database of the P. ginseng genome (http:/ / ginsengdb.snu.ac.kr, accessed on 20 January 2022), and the putative ORF sequence was verified by sequencing after subcloning.

The predicted amino acid sequence of $\mathrm{PgCYP703A4}$ was used to search for homologous proteins via National Center for Biotechnology Information-Basic Local Alignment Search Tool (NCBI-BLASTX, http:/ / www.ncbi.nlm.nih.gov/BLAST/, accessed on 18 January 2022). Sequence alignment was conducted using Clustal X V1.83, and a neighborjoining tree was constructed using the MEGA4 software V.4.0.1, with the reliability of each node established by the bootstrap method. The subcellular localization for the $\mathrm{N}$-terminus was predicted by PSORTdb (http://www.psort.org/psortb/, accessed on 18 January 2022) [16], and the hydropathy value was calculated using the previously described method [17]. The 1000-bp sequence upstream of the ATG-coding site in the genomic DNA sequence of $\mathrm{PgCYP703}$ were used as promoters to predict Cis-acting elements by New PLACE (http:/ / www.dna.affrc.go.jp/PLACE/?action=newplace, accessed on 20 December 2021) [18].

\subsection{Vector Construction and A. thaliana Transformation}

The full-length CYP703 gene was amplified from P. ginseng flower cDNA and cloned into the SalI and SpeI sites of the pCAMBIA1390 vector containing the Cauliflower Mosaic Virus $35 \mathrm{~S}$ promoter and yellow fluorescent protein. After nucleotide sequence verification, A. thaliana transformation was conducted using Agrobacterium tumefaciens C58C1 (pMP90) [19]. The insertion of transgenes into the transformants was confirmed via polymerase chain reaction (PCR). Heterozygous plants with a 3:1 segregation ratio on antibiotic plates which indicate the single gene insertion, were selected for additional analyses. Among the several T2 independent lines, two lines were selected for further statistical and metabolite analyses.

\subsection{Gene Expression Analysis}

Total RNA extraction from frozen samples was performed using the RNeasy Mini Kit (Qiagen, Valencia, CA, USA.), where 1- $\mu$ g of the total RNA was used as a reverse transcription template. For qRT-PCR, 100 -ng cDNA in a $10-\mu \mathrm{L}$ reaction volume and SYBR ${ }^{\circledR}$ Green Sensimix Plus Master Mix (Quantace, Watford, England) were used. Specific primers for $P g-$ CYP703A4 (F-5'-CTACGGGTGCAATGATGTTG-3' and R-5' - TGCATGGAAAACGACTCA AG- $3^{\prime}$ ) and a constitutively expressed $P$. ginseng actin gene (forward, $5^{\prime}$-AGAGATTCCGCTGT CCAGAA- $3^{\prime}$ and reverse, $5^{\prime}$-ATCAGCGATACCAGGGAACA- $3^{\prime}$ ) or $A$. thaliana actin gene (forward, $5^{\prime}$-GTGTGTCTTGTCTTATCTGGTTCG- $3^{\prime}$ and reverse $5^{\prime}$-AATAGCTGCATTGT CACCCGATACT- $3^{\prime}$ ) were used as an internal reference. qRT-PCR was conducted using a CFX Connect Real-Time PCR Detection System (BIO-RAD, Hercules, CA, USA) with the following program: $30 \mathrm{~s}$ at $95^{\circ} \mathrm{C}$, followed by 40 cycles of $95{ }^{\circ} \mathrm{C}$ for $3 \mathrm{~s}$ and $60{ }^{\circ} \mathrm{C}$ for $20 \mathrm{~s}$. The threshold cycle $(\mathrm{Ct})$ reflects the number of cycles where the fluorescence intensity at the original exponential stage of PCR amplification was significantly greater than the background fluorescence. To determine the relative fold differences in template abundance for each sample, the $\mathrm{Ct}$ value for PgCYP703A4 was normalized to the $\mathrm{Ct}$ value for $\beta$-actin and calculated relative to a calibrator using the formula $2^{-\Delta \Delta C t}$. Each qRT-PCR was technically repeated at least three times. Spatial expression of PgCYP703 transcript in ginseng anther was analyzed by In situ hybridization, as reported previously [12]. 


\subsection{Histological Analysis}

Semi-thin sectioning was performed using anthers of mature flowers from 4-weekold plants. After fixing in FAA, the samples were dehydrated with an ethanol gradient (70\%, 80\%, 90\%, and 100\%) allowing 30 min for each step. Samples were then embedded in KULZER Technovit 7100 cold polymerizing resin by pre-infiltration, infiltration, and embedding at $45^{\circ} \mathrm{C}$, according to the previously described methods [20]. Samples were sectioned to a thickness of $4 \mu \mathrm{m}$ in an Ultratome III ultramicrotome (LBK) and stained with $0.25 \%$ toluidine blue O (Chroma Gesellshaft Shaud). Bright-field photographs of the anther and silique sections were obtained using a Nikon ECLIPSE 80i microscope.

Scanning electron microscopy (SEM) was employed to analyze mature anthers that were fixed in FAA and dehydrated using $20-100 \%$ ethanol (10\% increments) allowing $3 \mathrm{~min}$ for each step. The samples were then dried at the critical point temperature (Leica EM CPD300). A 5-nm-thick Aurum coating was paced on the samples with a Leica EM SCD050 ion sputter. The Aurum-coated samples were observed using a Hitachi S3400N SEM.

\subsection{Analysis of Silique Fatty Acids}

Cutin from the siliques of 5-week-old plants were examined as previously described [15,21]. Dried siliques (10-20 mg) were extracted in $2 \mathrm{~mL}$ of chloroform and spiked with $10 \mu \mathrm{g}$ of tetracosane (Fluka) as the internal standard. Solvent was evaporated under a light stream of nitrogen, and the compounds containing free hydroxyl and carboxyl groups were transformed to trimethysilyl ethers and esters using $20-\mu \mathrm{L}$ bis- $(\mathrm{N}$, $\mathrm{N}$-trimethysilyl)-trifluoroacetamine (Sigma-Aldrich, St. Louis, MO, USA) in $20-\mu \mathrm{L}$ pyridine for $40 \mathrm{~min}$ at $70^{\circ} \mathrm{C}$. The monomers were identified from their electron ionization-mass spectrometry spectra (70 eV, m/z 50 to 700) after GC separation (column $30 \mathrm{~mm} \times 0.32 \mathrm{~mm}$ $\times 0.1 \mu \mathrm{m}$ film thickness [DB-1; J\&W Scientific]). Gas chromatography-mass spectrometry (GC-MS) (Agilent gas chromatograph coupled with an Agilent 5973N quadrupole mass selective detector) and gas chromatography-flame ionization detection (GC-FID) (Agilent 6890 gas chromatograph) analyses were conducted. The means of three independent replicates were statistically analyzed and compared with control $(* p<0.05)$ using Student's $t$-test.

\section{Results}

\subsection{Identification of CYP703 Gene in P. ginseng}

CYP families often have many paralogs, but the CYP703 family was reported to be a single-gene-member family [8]. Analysis of the P. ginseng genome scaffold and CDS revealed that $P$. ginseng contains two scaffold sequences with high similarity to CYP703. Among the two scaffolds, scaffold 1562 contained a full-length CDS of CYP703 (Pg_S1562.26), which contained a two-exons and one-intron structure (Figure 1), similar to the $A$. thaliana gene structure. On the contrary, two CDS sequences, S6323.1 and S6323.2, present in scaffold 6323 were partial sequences of CYP703. Therefore, we concluded that the $P$. ginseng genome encodes just one PgCYP703 member, whereas the others are nonfunctional genes. The recent genome duplications of the $P$. ginseng genome [22] might explain the presence of two genes that can duplicated, in which one of the gene has retained the original sequence and function, whereas the other became a pseudogene. Similarly, CYP703A was noted as a single functional sequence in the poplar genome (Populus trichocarpa) [8]. 
A

Pg_S1562.26 ATG $\quad 1119 \mathrm{bp} \quad$ TGA

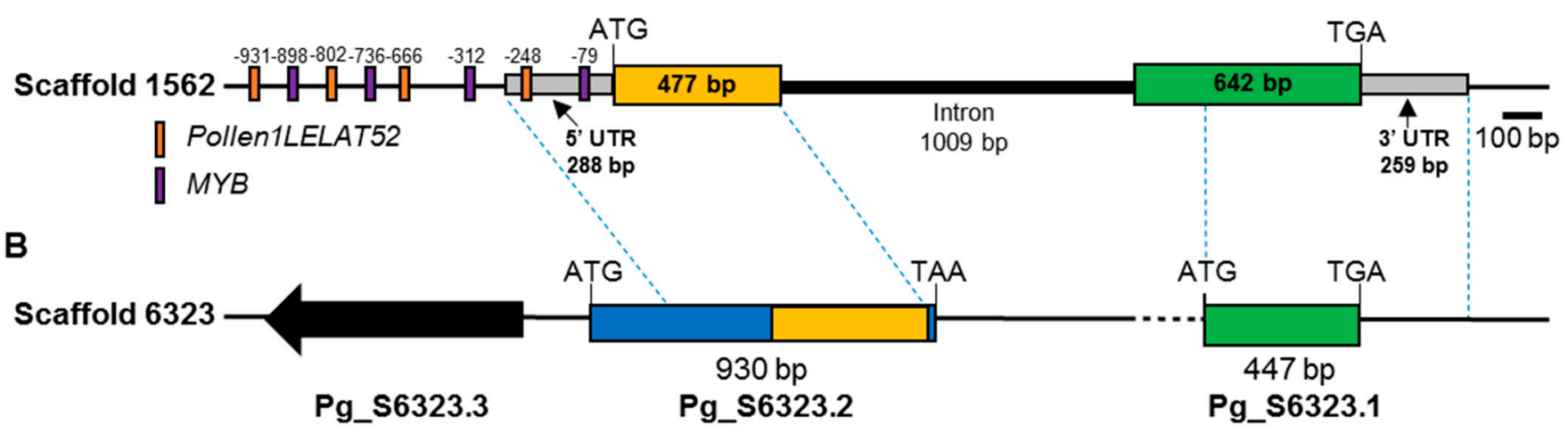

Figure 1. Analysis of gene and promoter structure of PgCYP703A4 and its pseudogene. Genomic sequence of scaffolds containing PgCYP703A4 and similar sequences were identified from the P. ginseng genome database (http:/ / ginsengdb.snu.ac.kr/ accessed on 20 January 2022). (A) PgCYP703A4 gene was confimed as Pg_S1562.26 CDS, which encoded on Scaffold 1562. The coding regions (orange and green boxes) are interrupted by 1009 base pair (bp) intron. The upstream 1000-bp region from the translation start site has four POLLEN1LELAT52 binding-predicted sites and four MYBCORE bindingpredicted sites. (B) A similar sequence structure was identified from Pg_scaffold6323 encoding two CDSs, assumed to be pseudogenes. The transcript was separated into two partial CDS sequences (Pg_S6323.2 and Pg_S6323.1). Dashed line indicates closed sequences between two scaffolds.

A putative ORF sequence, which had a length of $1119 \mathrm{bp}$ and encoded 372 amino acids (Figure 1), was verified by sequencing, and an NCBI-BLAST search displayed the conserved superfamily CYP. There are three functionally reported genes: two CYP703A members registered in the Plant P450 Database (http:/ / erda.dk/public/vgrid/PlantP450/, accessed on 10 December 2021) (CYP703A1 from a Petunia hybrida [23] and AtCYP703A2 [8]) and O. sativa CYP703A3 [7]. We named the CYP703 gene identified in P. ginseng as PgCYP703A4.

\subsection{Sequence Alignment and Phylogenetic Analysis}

CYP703 enzymes belong to the CYP71 clan, which includes the diverse families of P450 in plants $[4,7]$. To obtain information about the potential functions and evolutionary roles of $\mathrm{PgCY}$ P703A4, the full-length protein was used as a query to search for homologs in NCBI databases and the The $A$. thaliana Information Resource using NCBI-BLASTX. Highly similar homologs of PgCYP703A4 were detected in various dicot plant species whose genome sequences were available, although functional studies were limited. To see similarity of amino acids in various plant species, we selected the 10 closest sequences, and representative CYP71 members from $A$. thaliana were used to create a phylogenetic tree (Figure 2). Based on our phylogenetic comparison, $\mathrm{PgCYP703A4}$ was placed in subfamily CYP703, separate from the other subfamilies of the CYP71 clan; CYP98, CYP73, CYP78, CYP84, CYP82, CYP81, CYP76, CYP61, CYP83, CYP705, and CYP701. CYP78 members hydoxylate short-chain fatty acids [24], CYP73 family members hydroxylate cinnamic acid [25], and CYP84 members are involved in lignin and flavonoid synthesis [26], thus indicating CYP71 clan subfamilies are not specific, rather showed various functions on metabolites. 


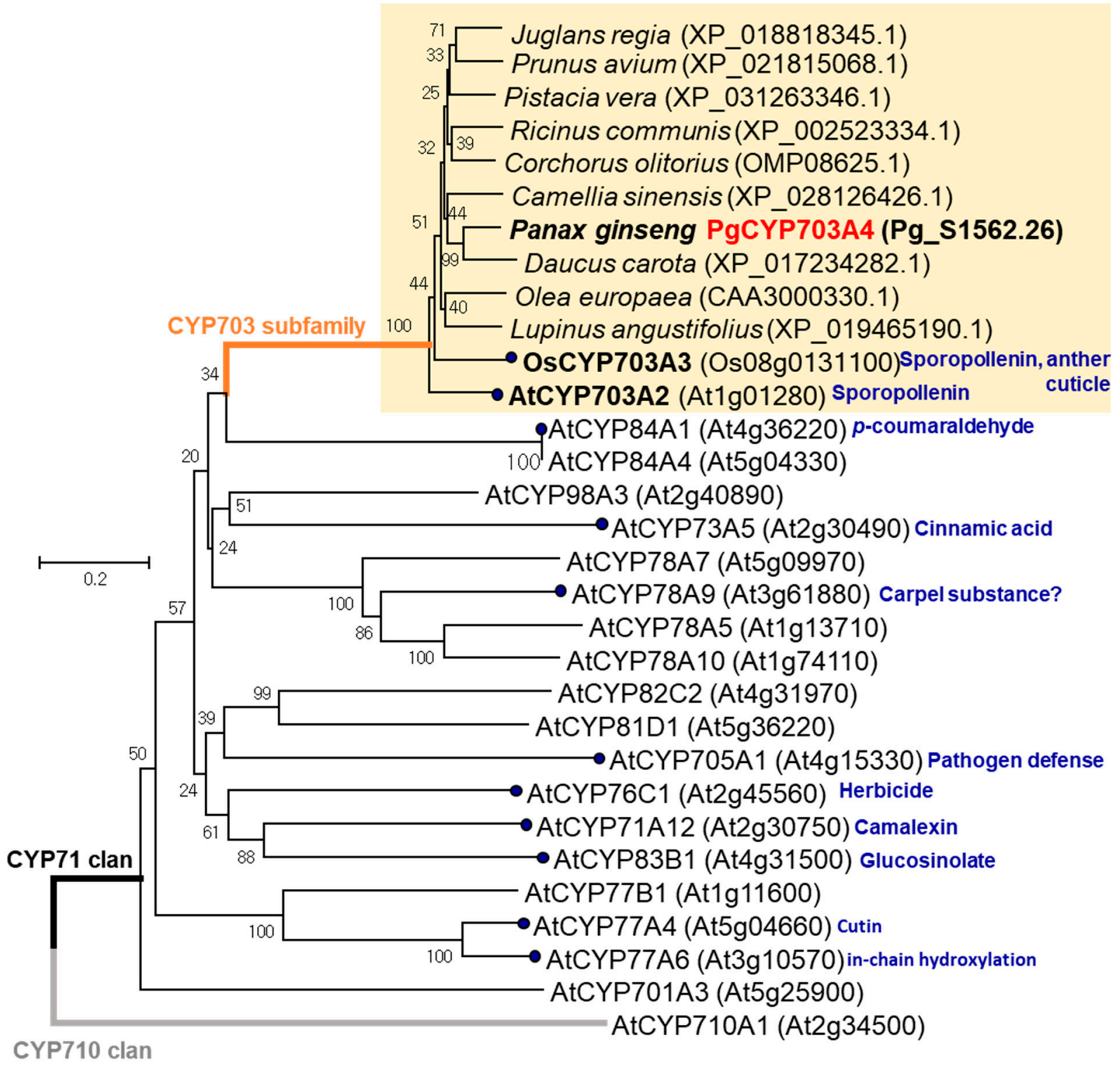

Figure 2. Phylogenetic analysis of PgCYP703. Neighbor-joining method analysis was conducted using the full-length amino acid sequences of PgCYP703A4 and closely related CYP703 subfamily members (Figure S1), in addition to the A. thaliana members of the CYP71 clan and outgroup of the CYP710 clan. The scale bar shows 0.2 amino acid substitutions per site. The reported CYP703A genes are distinguished by bold font with black dots. CYP703 subfamilies of the CYP71 clan are indicated by the yellow box. The functionally reported genes are indicated by circles and brief role in the right side. In figure, 'At' means Arabidopsis thaliana's protein and 'Os' means Oryza sativa's protein, and other plants are presented with full scientific name. NCBI accession numbers for other species and annotation numbers for $P$. ginseng, $A$. thalina and $O$. sativa species are indicated inside bracket.

Multiple sequence alignment (Figure S1) revealed that the CYP703 proteins and PgCYP703A4 contain the conserved domains of the axial ligand for heme, the I-helix involved in oxygen binding, the Arg of the "PERF" consensus, and the E-R-R of the K-helix consensus (KETLR). Of these domains, only the cysteine of the heme-binding domain and the E-R-R triad are conserved in all plant cytochrome P450 sequences $[27,28]$. CYP703 members have a Phe-His substitution in the $\mathrm{P}(\mathrm{E}) \mathrm{R}(\mathrm{F})$ domain of CYP, known as a clade signature of the CYP703 family [28]. In addition, CYP703 members have an A-A-T-D-T-S motif (Figure S1) in the A/G-G-X-E/D-T-T/S domain, which is involved in oxygen binding 
and activation [28]. Of the subfamilies in the CYP71 clan, substitution of A for the second $\mathrm{G}$ is unique to the CYP703 subfamily.

Plant P450s are usually bound to membranes, anchoring to the cytoplasmic surface of the endoplasmic reticulum (ER) through a short hydrophobic segment of their N-terminus [29] The predicted transit peptide of PgCYP703A4 (indicated arrow in Figure S1) was shown to be positioned at its N-terminus with a cytoplasmic location [16] targeting ER with 69\% certainty, predicted by PSORT (Prediction of Protein Localization Sites, version 6.4) Prediction program. Fatty acids are hydroxylated in the ER of plant cells through members of the CYP family $[8,30,31]$. The PgCYP703 hydrophobicity profile and those of its closest homologs indicated that both the $\mathrm{N}$ - and C-terminal regions, as well as the CYP703 motifs, are highly conserved (Figure S2).

\subsection{Gene Expression Analysis of PgCYP703A4}

To verify the conserved function of $\mathrm{PgCYP703A4}$ in the male reproductive organ, as has been described for A. thaliana [8] and O. sativa [7], we conducted PgCYP704B1 expression analysis via qRT-PCR using $P$. ginseng tissues, such as the see, root, stem, leaf, flower buds, and fruit at different age. PgCYP703A4 was specifically expressed in flower buds (Figure 3A).

A

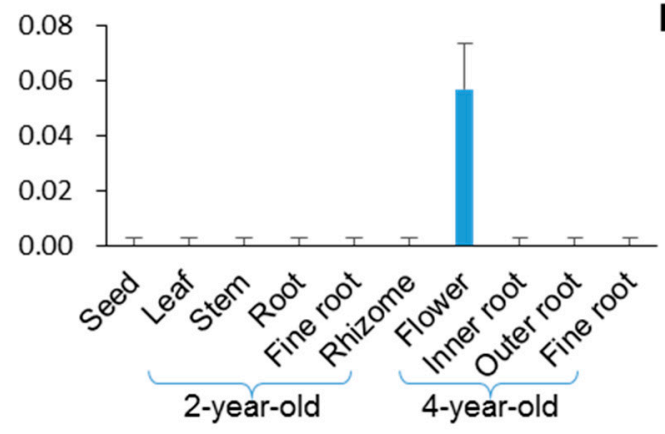

B

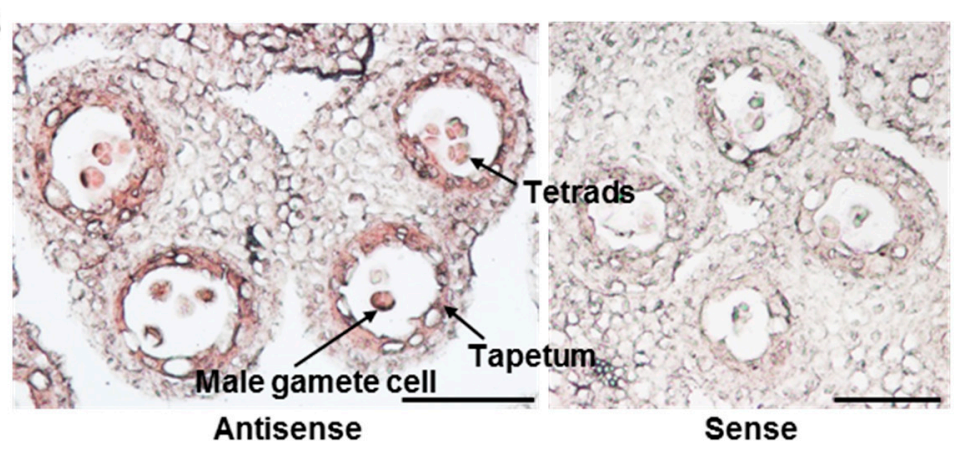

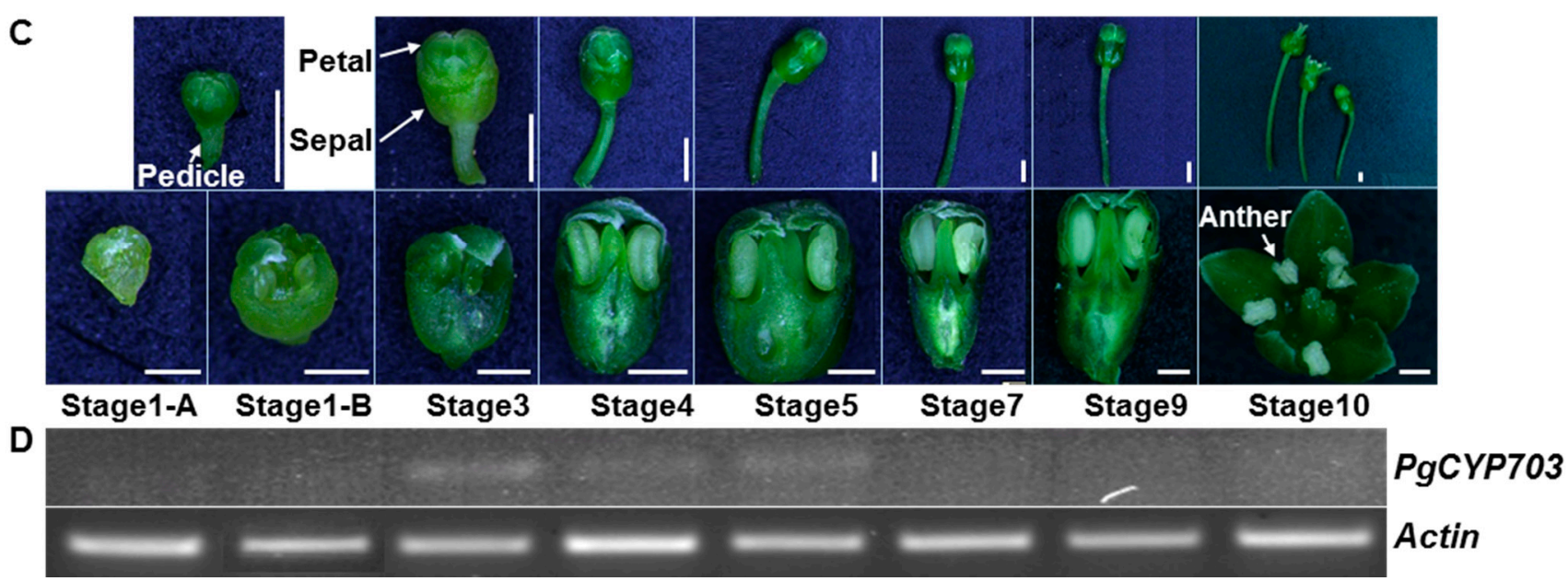

Figure 3. Tissue expression analysis of $P g C Y P 703 A 4$ in $P$. ginseng. (A) Quantitative expression analysis of $P g C Y P 703 A 4$ in various tissues at different age of $P$. ginseng plant. The expression levels were analyzed via realtime PCR, and PgActin served as the control. Values indicate mean of three technical replicates \pm SE. (B) In situ hybridization analysis of PgCYP703A4 in P. ginseng anther at stage 4 showing its expression (dark pink) in tapetal cells and microspores. Right image shows the anther with hybridized $P g C Y P 703 A 4$ sense probe, as control. Scale bars indicate $500 \mu \mathrm{m}$. (C) Flower tissues of the P. ginseng at anther developmental stages [14] were used for RT-PCR. The scale bars of uppder photo indicate $1 \mathrm{~mm}$, and lower photo indicate $500 \mu \mathrm{m}$. (D) RT-PCR gel images of PgCYP703A4 at seven stages of $P$. ginseng flowers show that PgCYP703A4 are expressed only during Stage 3 to 5. PgActin served as a control. 
In situ hybridization experiments revealed that $\mathrm{PgCYP703A4}$ was expressed in the tapetal cell layer and tetrad cells (meiocytes after the second meiosis) in the anther (Figure $3 \mathrm{~B}$ ). The tapetum, the innermost layer of anther wall, plays a crucial role in pollen development by nursing and releasing the microspore [32]. During the developmental stage of anther, the $P g$ CYP703A4 expression was observed in meiosis to form young microspores (Figure 3C,D) [13].

To illuminate the regulation of gene expression, the 1000-bp upstream region from the coding sequence of PgCYP703A4 was analyzed for cis-elements (Figure 1). The promoters contained various elements. Among them, we noted POLLEN1LELAT52 and MYBCORE, known as regulatory factors that are related to pollen and early pollen development, respectively $[33,34]$. These results indicated that MYBCORE and POLLEN1LELAT52 might bind to the promoters of $P g C Y P 703$, which leads to their specific expression during anther development.

\subsection{Phenotype Analysis of PgCYP3A4 Overexpressing A. thaliana}

Due to difficulties in obtaining transgenic regenerated $P$. ginseng plants, we generated PgCYP703A4 overexpressing transgenic Arabidopsis ( $P g C Y P 703 A 40 x)$ to examine its functional role in planta (Figure 4A-D). The stable incorporation of the $P g C Y P 703 A 4$ gene and its heteroexpression was confirmed via RT-PCR (Figure 4B). PgCYP703A4ox produced slightly taller plants compared with the wild type, but not significant (Figure 4A). Notably, the siliques increased in size by $20 \%$ compared with the wild type and PgCYP703A4ox siliuqes contain higher number of seeds than wile type significantly (Figure 4C,D).
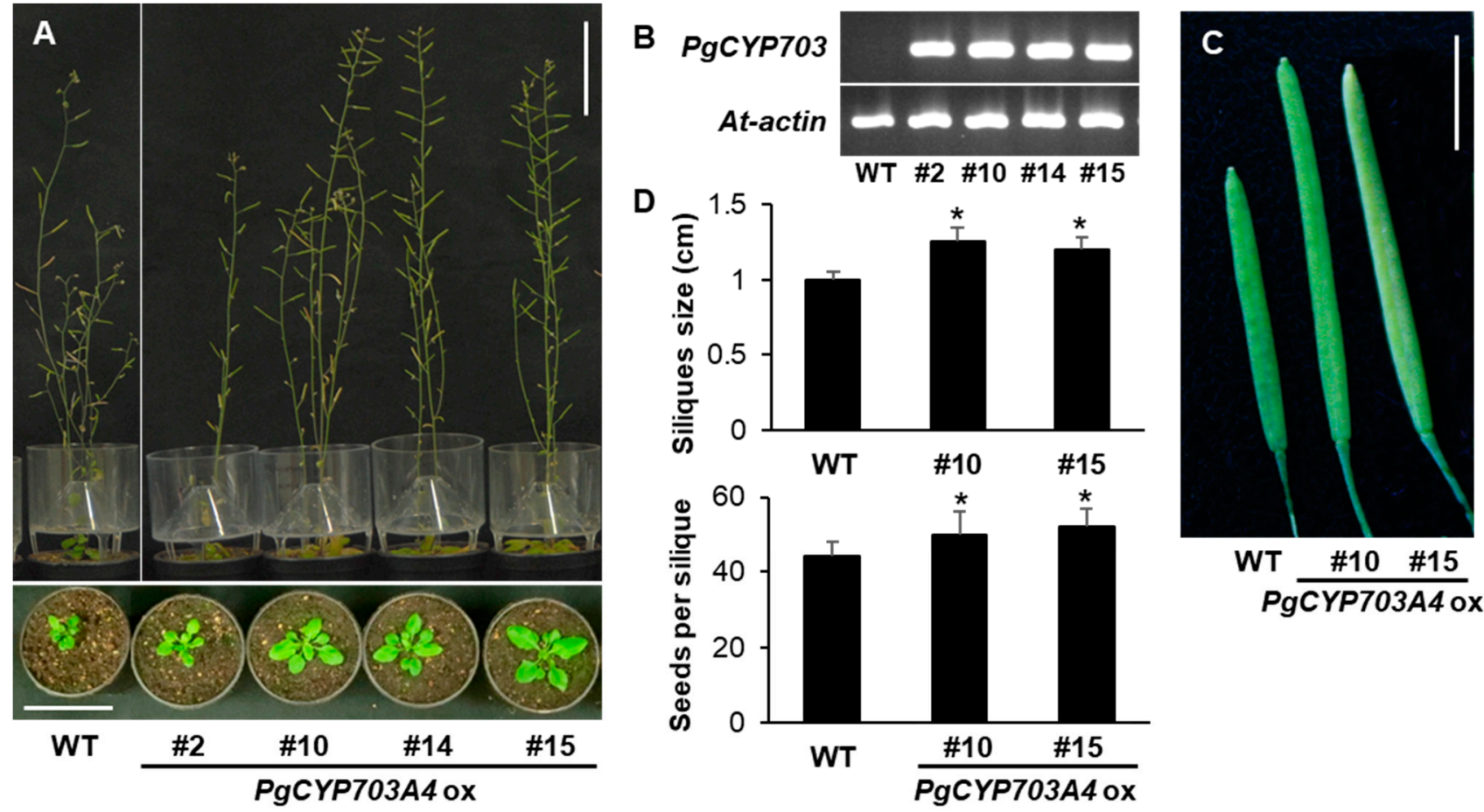

Figure 4. Phenotype analysis of PgCYP703A4 overexpressing A. thaliana. (A) Growth phenotype of four different PgCYP703A4 overexpression lines and wild type at 2 week- and 7 week- old. Scale bar indicates $5 \mathrm{~cm}$. (B) Detection of PgCYP703A4 transcription in transgenic A. thaliana's rosette leaves. At actin served as the control. (C,D) Silique size of PgCYP703A4 overexpression lines. Scale bar indicates $5 \mathrm{~mm}$. Values indicate mean of 20 biological replicates \pm SD. ${ }^{*} p<0.05$.

A. thaliana CYP703A3 mutant was reported to show impaired pollen walls lacking a normal exine layer, which leads to partial male sterility [8]. To determine how PgCYP703A4 affects pollen wall formation, we observed the anthers and pollen phenotype by semi-thin cross-section and SEM (Figure S3). The anther, pollen, and pistil of PgCYP703A4ox appeared similar to the wild type, whereas CYP703A3 exhibited aborted pollen without outer elegant 
wall formation (Figure S3). Therefore, pollen viability and reproductive organ function was not altered by $\mathrm{PgCYP703A4}$ gene overexpression.

\subsection{Fatty Acid Composition of PgCYP703A4-Overexpressing A. thaliana}

The cuticle, a hydrophobic layer that coats the surface of the aerial organs, such as leaves, stems, flowers, and fruits, is a biopolymer that is composed of two classes of lipophilic constituents, namely, cutin and waxes $[35,36]$. Since the silique phenotype of $\mathrm{PgCYP703A40x}$ is elongated and the exocarp is made of cutin, we further conducted GC-MS and GC-FID.

The overall sum of cutin monomers were not significantly different between wild type and PgCYP703ox lines. We found that two independent lines alter the composition of fatty acids, with some variation, which might explain complex fatty acids metabolism for the cutin polymer. For example, compared with the wild type, the PgCYP703A4ox \#10 significantly increased saturated fatty acids of C18:0, C18:1, C26:1 and C28:0, (Figure 5A), dicarboxylic fatty acids of C18:0, C22:0, C24:0, and C26:0 (Figure 5C) and terminal-hydroxy fatty acids of C18:3, C22:0, and C24:0 (Figure 5D), and C28:0 alcohol type (Figure 5E). PgCYP703A4ox \#15 increased C24:0, C25:0, and C26:0 saturated fatty acids (Figure 5A), and 2-hydroxyl fatty acids, such as C16:0, C23:0, C24:0, C25:0, C26:0 (Figure 5B). The dicarboxylic fatty acids of C22:0 and C24:0 were significantly increased in the PgCYP703A4ox lines (Figure 5C). The levels of the terminal-hydroxy fatty acid C22:0 was found to increase up to two times in PgCYP703A4ox in comparison with the wild type (Figure 5D). These data indicate that the $P g C Y P 703 A 4$ overexpression in $A$. thaliana affects the fatty acid composition of cutin monomers in siliques.

A

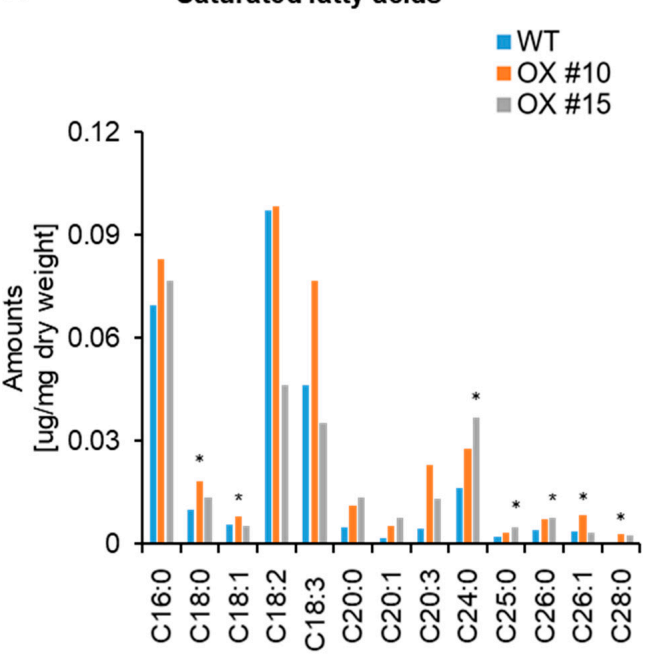

D

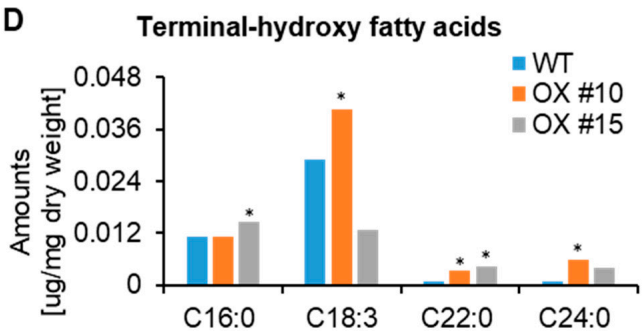

B

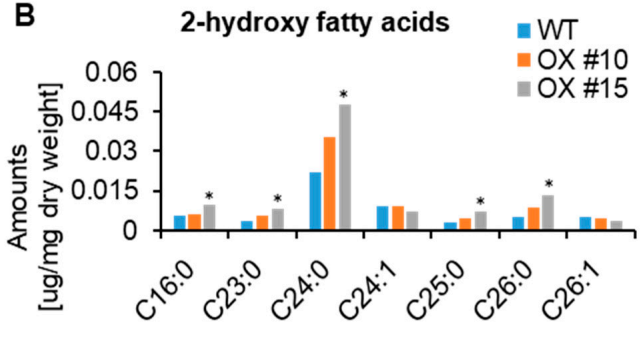

C Dicarboxylic fatty acids

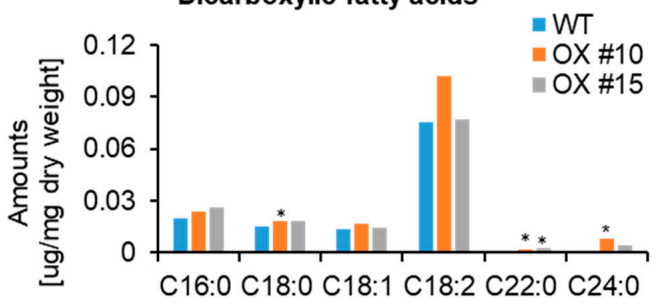

E

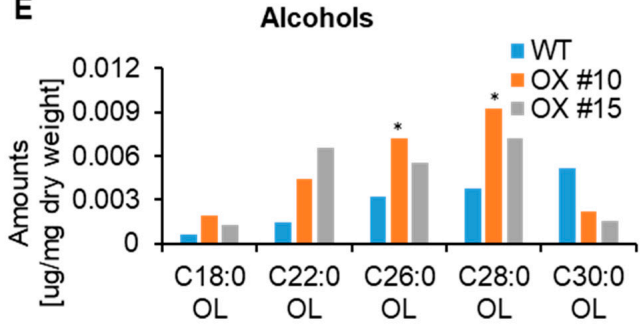

Figure 5. Chemical analysis of silique cutin monomers in wild type and PgCYP703ox lines via GC-MS and GC-FID. (A) Saturated fatty acids per milligram of dry weight ( $\mu \mathrm{g} / \mathrm{mg}$ ). (B) 2-hydroxy fatty acids per milligram of dry weight (ug/mg). (C) Dicarboxylic fatty acids per milligram of dry weight (ug/mg). (D) Terminal-hydroxy fatty acids per milligram of dry weight (ug/mg). (E) Alcohols per milligram of dry weight $(\mathrm{ug} / \mathrm{mg})$. Values indicate mean of five biological replicates $\pm \mathrm{SD} .{ }^{*} p<0.05$. 


\section{Discussion}

Plants have evolved a variety of enzymes for the in-chain $\alpha-, \beta-$-, and $\omega$-hydroxylation of fatty acids. Hydroxylated fatty acids are the biosynthetic intermediates of plant biopolymers, such as cutin and suberin, which make up the barriers from land plant stress situations. Thus, fatty acid metabolic enzymes are critical for plants; however, the role of CYP members in controlling the development of $P$. ginseng has not been well studied. In this study, we characterized $P g C Y P 703 A 4$ and its role in the fatty acid metabolism. The $\mathrm{PgCYP703A4}$ expression only detected at the flowering stage during microspore formation in the tapetum and gamete cells, which are active in sporopollenin synthesis. The overexpression of $P g C Y P 703 A 4$ in A. thaliana increased silique size and seed production without affecting gamete cell, similar to $P g C Y P 704 B 1$ [15].

CYPs constitute the largest family of enzymes in plant metabolism and represent plant evolution in terms of plant metabolism in development and adaptation, such as signaling, defense, and polymerization of complex chemical substances [37]. Among the 11 land plant clans, the CYP71 clan represents more than half of all CYPs in higher plants; consequently, a wide diversity of functions makes them more difficult to predict their preferred substrates than other clans [37]. In addition to CYP703, CYP77 family members, AtCYP77A4 and AtCYP77A6, can in-chain-hydroxylate fatty acids to form precursors of cutin $[9,38,39]$. Looking at their phylogenetic relationship, CYP703 diversified prior to the emergence of CYP77s as spore protectors. CYP703 is an ancient gene family that is required for land plants, whereas CYP77 is required in only angiosperms [37]. However, both CYP703A and CYP77A function as in-chain hydroxylases, compared with most other enzymes that are end-chain $(\omega)$ hydroxylase [30].

In addition, a part of the CYP71 clan, the CYP78 subfamily genes exhibited fatty acid hydroxylation reactions, particularly for short chains [24]. Different from a single member of CYP703 (Figure 2), the CYP78 family contains several members in A. thaliana (Figure 2), indicating late gene duplication for the species. CYP78A family members regulate reproductive organ development but are more related to female organs. A. thaliana gene CYP78A9 was reported to be involved in the control of carpel shape [40]. The overexpression of CYP78A9 results in large, seedless fruit, although the metabolites have not been discovered [40]. O. sativa gene OsCYP78A13 promotes seed growth by regulating the embryo and endosperm size, as well as spikelet hull development [41]. PaCYP78A9 regulates fruit size in Prunus avium, showing increases in silique and seed size in $A$. thaliana by hetero-overexpression [42]. In addition to the above studies of the CYP71 clan other functions include glucosinolate production [43], p-coumaraldehyde hydroxylation [44], and pathogen defense function [45], as indicated in Figure 2. It is clear that the CYP71 clan has a large diversity of functions, but only CYP703 and CYP78 families of this clan, have the conserved PERF consensus, and both subfamiles are involved in plant reproductive development. Further studies are required to identify its positive relationship with biological function.

In $P$. ginseng, reproductive development and functional studies are scarce. We previously identified a functional ortholog of AtCYP704B1, termed PgCYP704B1 [15]. The CYP704B family, which belongs to the CYP86 clan, is involved in the $\omega$-hydroxylation of long-chain fatty acids. Altered exine in the pollen wall was detected in mutant of $A$. thaliana cyp704B1 [46], Brassica napus CYP704B1 [47], and O. sativa CYP704B2 [36]. However, O. sativa CYP704B2 also had an undeveloped anther cuticle and sterile male phenotype [36]. It is similar to CYP703A, although it is in a separate clan. Similarly, CYP701A and CYP88A, which belong to the CYP71 and CYP85 clans, respectively, act sequentially in the same pathway as ent-kaurene oxidase and kaurenolic acid oxidase, respectively [48]. With the early evolution of CYPs, CYP703 and CYP704 could be involved in cutin biopolymer synthesis, particularly for pollen wall polymers, for in-chain and $\omega$-hydroxylation, respectively. This study was limited by the difficulty of obtaining flowers from transgenic P. ginseng. However, further studies on $P$. ginseng development is required to develop hybrid and male sterile system for breeding. 
Taken together, $P g C Y P 703 A 4$, a member of CYP703A in the CYP71 clan, and $P g-$ CYP704B1 [22], in the CYP86 clan, are similarly expressed in the $P$. ginseng tapetum and meiotic cells, and overexpression in A. thaliana affects fatty acid metabolism in siliques. Previous studies on A. thaliana and O. sativa examined knockout mutants displaying partial or full male sterility $[7,9,36,46]$ and therefore did not further investigate the phenotype regarding fruit development. This requires further investigation to determine the role of hydroxylated fatty acids in sporopollenin synthesis and the development of the silique cuticle.

Supplementary Materials: The following are available online at https://www.mdpi.com/article/10 .3390 / plants11030383/s1, Figure S1: Multiple alignment of PgCYP703 with homologous proteins from other plant species. Figure S2: Superimposed hydrophobicity profile predictions for PgCYP703A4 and selected homologs from the 703A4 family. Figure S3: Cytological analysis of an Arabidopsis mutant and the PgCYP703A4 overexpression line.

Author Contributions: Y.-J.K. and D.Z. conceived and design the project and experiments. J.S. (Jeniffer Silva) and J.K. conducted the experiments and data arrangements. C.P., Y.K., N.P. and J.S. (Johan Sukweenadhi) contributed for bioinformatic analysis and plant growth. C.-O.H. and K.K.K. provide critical comments for metabolite analysis. J.Y. contributed for cytological analysis and J.S. (Jianxin Shi) performed the wax and cutin analysis. H.-J.S., H.C.P. and K.M.L. contributed for manuscript revision. Y.-J.K. and J.K. co-write the manuscript. All authors have read and agreed to the published version of the manuscript.

Funding: This work was supported by a 2-Year Research Grant of Pusan National University.

Institutional Review Board Statement: Not applicable.

Informed Consent Statement: Not applicable.

Data Availability Statement: Not applicable.

Conflicts of Interest: The authors declare no conflict of interest.

\section{References}

1. Nelson, D.R. Cytochrome P450 and the individuality of species. Arch. Biochem. Biophys. 1999, 369, 1-10. [CrossRef] [PubMed]

2. Chapple, C. Molecular-genetic analysis of plant cytochrome P450-dependent monooxygenases. Annu Rev. Plant Biol. 1998, 49, 311-343. [CrossRef] [PubMed]

3. Mizutani, M.; Ohta, D. Diversification of P450 genes during land plant evolution. Annu Rev. Pland Biol. 2010, 61, 291-315. [CrossRef] [PubMed]

4. Nelson, D.R.; Schuler, M.A.; Paquette, S.M.; Reichhart, W.R.; Bak, S. Comparative genomics of rice and Arabidopsis. Analysis of 727 cytochrome P450 genes and pseudogenes from a monocot and a dicot. Plant Physiol. 2004, 135, 756-772. [CrossRef] [PubMed]

5. Torutaeva, E.; Oh, S.A.; Park, S.K. Identification of new mutant alleles of Augmin subunits broadens spectrum of Augmin function during sexual reproduction in Arabidopsis. J. Plant Biol. 2020, 63, 485-494. [CrossRef]

6. Cui, X.; Wang, Q.; Yin, W.; Xu, H.; Wilson, Z.A.; Wei, C.; Pan, S.; Zhang, D. PMRD: A curated database for genes and mutants involved in plant male reproduction. BMC Plant Biol. 2012, 12, 215. [CrossRef]

7. Yang, X.; Wu, D.; Shi, J.; He, Y.; Pinot, F.; Grausem, B.; Yin, C.; Zhu, L.; Chen, M.; Luo, Z.; et al. Rice CYP703A3, a cytochrome P450 hydroxylase, is essential for development of anther cuticle and pollen exine. J. Integr. Plant Biol. 2014, 56, 979-994. [CrossRef]

8. Morant, M.; Jørgensen, K.; Schaller, H.; Pinot, F.; Møller, B.L.; Reichhart, D.W.; Bak, S. CYP703 is an ancient cytochrome P450 in land plants catalyzing in-chain hydroxylation of lauric acid to provide building blocks for sporopollenin synthesis in pollen. Plant Cell 2007, 19, 1473-1487. [CrossRef]

9. Li-Beisson, Y.; Pollard, M.; Sauveplane, V.; Pinot, F.; Ohlrogge, J.; Beisson, F. Nanoridges that characterize the surface morphology of flowers require the synthesis of cutin polyester. Proc. Natl. Acad. Sci. USA 2009, 51, 22008-22013. [CrossRef]

10. Torres, M.F.; Mathew, L.S.; Ahmed, I.; Al-Azwani, L.K.; Krueger, R.; Nuñez, D.R.; Mohamoud, Y.A.; Clark, A.G.; Suhre, K.; Malek, J.A. Genus-wide sequencing supports a two-locus model for sex-determination in Phoenix. Nat. Commun. 2018, 9, 3969. [CrossRef]

11. Kim, Y.J.; Zhang, D.; Yang, D.C. Biosynthesis and biotechnological production of ginsenosides. Biotechnol. Adv. 2015, 33, 717-735. [CrossRef] [PubMed]

12. Kim, Y.J.; Jang, M.G.; Zhu, L.; Silva, J.; Zhu, X.; Sukweenadhi, J.; Kwon, W.S.; Yang, D.C.; Zhang, D. Cytological characterization of anther development in Panax ginseng Meyer. Protoplasma 2015, 253, 1111-1124. [CrossRef] [PubMed]

13. Kim, Y.J.; Silva, J.; Zhang, D.; Shi, J.; Joo, S.C.; Jang, M.G.; Kwon, W.S.; Yang, D.C. Development of interspecies hybrids to increase ginseng biomass and ginsenoside yield. Plant Cell Rep. 2016, 36, 779-790. [CrossRef] [PubMed]

14. Silva, J.; Kim, Y.J.; Xiao, D.; Sukweenadhi, J.; Hu, T.; Kwon, W.S.; Hu, J.; Yang, D.C.; Zhang, D. Cytological analysis of ginseng carpel development. Protoplasma 2017, 254, 1909-1922. [CrossRef] [PubMed] 
15. Silva, J.; Sukweenadhi, J.; Myagmarjav, D.; Mohanan, P.; Yu, J.; Shi, J.; Jung, K.H.; Zhang, D.; Yang, D.C.; Kim, Y.J. Overexpression of a novel cytochrome P450 monooxygenase gene, CYP704B1, from Panax ginseng increase biomass of reproductive tissues in transgenic Arabidopsis. Mol. Biol. Rep. 2020, 47, 4507-4518. [CrossRef] [PubMed]

16. Yu, N.Y.; Wagner, J.R.; Laird, M.R.; Melli, G.; Rey, S.; Lo, R.; Dao, P.; Sahinalp, S.C.; Ester, M.; Foster, L.J.; et al. PSORTb 3.0: Improved protein subcellular localization prediction with refned localization subcategories and predictive capabilities for all prokaryotes. Bioinformatics 2010, 26, 1608-1615. [CrossRef]

17. Kyte, J.; Doolittle, R.F. A simple method for displaying the hydropathic character of a protein. J. Mol. Biol. 1982, 157, 105-132 [CrossRef]

18. Prestridge, D.S. SIGNAL SCAN: A computer program that scans DNA sequences for eukaryotic transcriptional elements Bioinformatics 1991, 7, 203-206. [CrossRef]

19. Betchtold, N.; Pelletier, G. In planta Agrobacterium-mediated transformation of adult Arabidopsis thaliana plants by vacuum infiltration. Methods Mol. Biol. 1998, 82, 256-266.

20. Zhang, Y.; Liang, W.; Shi, J.; Xu, J.; Zhang, D. MYB56 encoding a R2R3 MYB transcription factor regulates seed size in Arabidopsis thaliana. J. Integr. Plant Biol. 2013, 55, 1166-1178. [CrossRef]

21. Jung, K.H.; Han, M.J.; Lee, D.Y.; Lee, Y.S.; Schreiber, L.; Franke, R.; Faust, A.; Yephremov, A.; Saedler, H.; Kim, Y.W.; et al Wax-deficient anther1 is involved in cuticle and wax production in rice anther walls and is required for pollen development. Plant Cell 2006, 18, 3015-3032. [CrossRef]

22. Riederer, M.; Müller, C. Biology of the Plant Cuticle; Blackwell: Oxford, UK, 2006.

23. Imaishi, H.; Matsumoto, Y.; Ishitobi, U.; Ohkawa, H. Encoding of a cytochrome P450-dependent lauric acid monooxygenase by CYP703A1 specifically expressed in the floral buds of Petunia hybrida. Biosci. Biotechnol. Biochem. 1999, 63, 2082-2090. [CrossRef] [PubMed]

24. Kai, K.; Hashidzume, H.; Yoshimura, K.; Suzuki, H.; Sakurai, N.; Shibata, D.; Ohta, D. Metabolomics for the characterization of cytochromes P450-dependent fatty acid hydroxylation reactions in Arabidopsis. Plant Biotechnol. 2009, 26, 175-182. [CrossRef]

25. Pan, Y.; Michael, T.P.; Hudson, M.E.; Kay, S.A.; Chory, J.; Schuler, M.A. Cytochrome P450 monooxygenases as reporters for circadian-regulated pathways. Plant Physiol. 2009, 150, 858-878. [CrossRef] [PubMed]

26. Humphreys, J.M.; Hemm, M.R.; Chapple, C. New routes for lignin biosynthesis defined by biochemical characterization of recombinant ferulate 5-hydroxylase, a multifunctional cytochrome P450-dependent monooxygenase. Proc. Natl. Acad. Sci. USA 1999, 96, 10045-10050. [CrossRef] [PubMed]

27. Hasemann, C.A.; Kurumbail, R.G.; Boddupalli, S.S.; Peterson, J.A.; Deisenhofer, J. Structure and function of cytochromes P450: A comparative analysis of three crystal structures. Structure 1995, 2, 41-62. [CrossRef]

28. Reichhart, D.W.; Bak, S.; Paquette, S. Cytochromes P450. Arab. Book 2002, 1, e0028. [CrossRef] [PubMed]

29. Williams, P.A.; Cosme, J.; Sridhar, V.; Johnson, E.F.; McRee, D.E. Mammalian microsomal Cytochrome P450 monooxygenase: Structure adaptation for membrane binding and functional diversity. Mol. Cell 2000, 5, 121-131. [CrossRef]

30. Cabello-Hurtado, F.; Batard, Y.; Salaün, J.P.; Durst, F.; Pinot, F.; Reichhart, D.W. Cloning, expression in yeast, and functional characterization of CYP81B1, a plant cytochrome P450 that catalyzes in-chain hydroxylation of fatty acids. J. Biol. Chem. 1998, 273, 7260-7267. [CrossRef]

31. Kandel, S.; Sauveplane, V.; Olry, A.; Diss, L.; Benveniste, I.; Pinot, F. Cytochrome P450-dependent fatty acid hydroxylases in plants. Phytochem. Rev. 2006, 5, 359-372. [CrossRef]

32. Moon, S.; Hong, W.J.; Kim, Y.J.; Chandran, A.K.N.; Gho, Y.S.; Yoo, Y.H.; Nguyen, V.N.T.; An, G.; Park, S.K.; Jung, K.H. Comparative transcriptome analysis reveals gene regulatory mechanism of UDT1 on anther development. J. Plant Biol. 2020, 63, 289-296. [CrossRef]

33. Li, J.; Yuan, J.; Li, M. Characterization of putative cis-regulatory elements in genes preferentially expressed in Arabidopsis male meiocytes. BioMed Res. Int. 2014, 708364, 10.

34. Nguyen, T.D.; Moon, S.; Nguyen, V.N.T.; Gho, Y.; Chandran, A.K.N.; Soh, M.S.; Song, J.T.; An, G.; Oh, S.A.; Park, S.K.; et al Genome-wide identification and analysis of rice genes preferentially expressed in pollen at an early developmental stage. Plant Mol. Biol. 2016, 92, 71-88. [CrossRef] [PubMed]

35. Kim, N.H.; Jayakodi, M.; Lee, S.C.; Choi, B.S.; Jang, W.; Lee, J.; Kim, H.H.; Waminal, N.E.; Lakshmanan, M.; Nguyen, B.V.; et al Genome and evolution of the shade-requiring medicinal herb Panax ginseng. Plant Biotechnol. J. 2018, 16, 1904-1917. [CrossRef] [PubMed]

36. Li, H.; Pinot, F.; Sauveplane, V.; Reichhart, D.W.; Diehl, P.; Schreiber, L.; Franke, R.; Zhang, P.; Chen, L.; Gao, Y.; et al. Cytochrome P450 family member CYP704B2 catalyzes the $\omega$-hydroxylation of fatty acids and is required for anther cutin biosynthesis and pollen exine formation in rice. Plant Cell 2010, 22, 173-190. [CrossRef]

37. Nelson, D.; Reichhart, D.W. A P450-centric view of plant evolution. Plant J. 2011, 66, 194-211. [CrossRef]

38. Sauveplane, V.; Kandel, S.; Kastner, P.E.; Ehlting, J.; Compagnon, V.; Werck- Reichhart, D.; Pinot, F. Arabidopsis thaliana CYP77A4 is the first cytochrome P450 able to catalyze the epoxidation of free fatty acids in plants. FEBS J. 2009, 276, 719-735. [CrossRef]

39. Pinot, F.; Beisson, F. Cytochrome P450 metabolizing fatty acids in plants: Characterization and physiological roles. FEBS J. 2011, 278, 195-205. [CrossRef]

40. Ito, T.; Meyerowitz, E.M. Overexpression of a gene encoding a cytochrome P450, CYP78A9, induces large and seedless fruit in Arabidopsis. Plant Cell 2000, 9, 1541-1550. [CrossRef] 
41. Xu, F.; Fang, J.; Ou, S.; Gao, S.; Zhang, F.; Du, L.; Xiao, Y.; Wang, H.; Sun, X.; Chu, J.; et al. Variations in CYP78A13 coding region influence grain size and yield in rice. Plant Cell 2015, 38, 800-811. [CrossRef]

42. Qi, X.; Liu, C.; Song, L.; Li, Y.; Li, M. PaCYP78A9, a Cytochrome P450, regulates fruit size in Sweet Cherry (Prunus avium L.). Front. Plant Sci. 2017, 8, 2076. [CrossRef] [PubMed]

43. Naur, P.; Petersen, B.L.; Mikkelsen, M.D.; Bak, S.; Rasmussen, H.; Olsen, C.E.; Halkier, B.A. CYP83A1 and CYP83B1, Two nonredundant cytochrome P450 enzymes metabolizing oximes in the biosynthesis of glucosinolates in Arabidopsis. Plant Physiol. 2003, 133, 63-72. [CrossRef] [PubMed]

44. Weng, J.K.; Li, X.; Stout, J.; Chapple, C. Independent origins of syringyl lignin in vascular plants. Proc. Natl. Acad. Sci. USA 2008, 105, 7887-7892. [CrossRef] [PubMed]

45. Sohrabi, R.; Huh, J.H.; Badieyan, S.; Rakotondraibe, L.H.; Kliebenstein, D.J.; Sobrado, P.; Tholl, D. In planta variation of volatile biosynthesis: An alternative biosynthetic route to the formation of the pathogen-induced volatile homoterpene DMNT via triterpene degradation in Arabidopsis roots. Plant Cell 2015, 27, 874-890. [CrossRef]

46. Dobritsa, A.A.; Shrestha, J.; Morant, M.; Pinot, F.; Matsuno, M.; Swanson, R.; Moller, B.L.; Preuss, D. CYP704B1 is a long-chain fatty acid omega-hydroxylase essential for sporopollenin synthesis in pollen of Arabidopsis. Plant Physiol. 2009, 151, 574-589. [CrossRef] [PubMed]

47. Yi, B.; Zeng, F.; Lei, S.; Chen, Y.; Yao, X.; Zhu, Y.; Wen, J.; Shen, J.; Ma, C.; Tu, J.; et al. Two duplicate CYP704B1-homologous genes BnMs1 and BnMs2 are required for pollen exine formation and tapetal development in Brassica napus. Plant J. 2010, 63, 925-938. [CrossRef]

48. Helliwell, C.A.; Chandler, P.M.; Poole, A.; Dennis, E.S.; Peacock, W.J. The CYP88A cytochrome P450, ent-kaurenoic acid oxidase, catalyzes three steps of the gibberellin biosynthesis pathway. Proc. Natl. Acad. Sci. USA 2001, 98, 2065-2070. [CrossRef] 